its name (see 'A culture of knowledge').

As for Eudid and Archimedes, there are 240 pages of Heath's editions with their erudite but dated commentaries. These may well help the reader with the mathematics, but historical scholarship has moved on. Unfortunately, the new comments are not much better. Each item is introduced with remarks that vary from the personal and insightful to the tired and incorrect. Among the latter is the supposed Greek crisis of the incommensurables, which was once presumed to have derailed the pythagoreans and which most historians these days think had little effect. The generally more accurate account of the life and work of Archimedes fails to mention the fact that the only manuscript of The Method - the most interesting of his works, in which he explained how he came to his discoveries - has recently re-entered the public domain after disappearing for most of the twentieth century.

Similar comments could be made about the more modern entries. These too are generally accurate but the origin of the information is not stated, so readers have no chance to catch up with contemporary scholarship in the history of mathematics. Nor can they find out how to sustain the flame of interest this book surely hopes to kindle, which is a pity, because Hawking's comments have an infectious enthusiasm for their subject and the book contains some great works.

Jeremy Gray is at the Centre for the History of the Mathematical Sciences, Faculty of Mathematics and Computing, OpenUniversity, Milton Keynes MK7 6AA, UK.

\title{
Sex and power
}

\section{Sexual Conflict \\ by Göran Arnqvist \& Locke Rowe \\ Princeton University Press: 2005. 360 pp. \\ $\$ 99.50, € 55$ (hbk); \$39.50, £26.95 (pbk)}

\section{Tracey Chapman}

Sexual conflict occurs because of the different evolutionary interests of males and females in reproductive decisions. Indeed, the differential investment of males and females in reproductive episodes and the low relatedness of mating partners make sexual conflict almost inevitable. Geoff Parker clearly laid out the theoretical basis for sexual conflict in the 1970 s, but it is only over the past ten or so years that a significant body of empirical work has started to emerge.

There has, however, been little agreement about what sexual conflict is, or what might constitute unambiguous evidence for it. In addition, the relative importance of sexual conflict in driving evolutionary change, and the extent to which it could contribute to reproductive isolation and speciation, are unknown. There is also considerable confusion about precisely how coevolution driven by sexual conflict is distinct from traditional models of coevolutionary change by sexual selection (that which arises from competition between individuals of the same sex for matings, or from mate choice).

Into this uncertainty comes the excellent and wide-ranging Sexual Conflict by Göran Arnqvist and Locke Rowe, the first booklength treatment of this emerging field. The authors have done a great service in defining the field and in illuminating some of its conceptual difficulties. They also bring together a rich treasury of examples that must surely stimulate discussion and further study.

The greatest strength of the book is in tackling the theory. Arnqvist and Rowe do a first-rate job of dissecting models of sexual selection and sexual conflict, and in getting to grips with the distinctions between them. The book is well worth reading for this alone. For example, the reader is led clearly through the idea that coevolution driven by sexual conflict is distinct from sexual selection: under sexual

IMAGE
UNAVAILABLE
FOR COPYRIGHT
REASONS

Sexual partners often seem to be pulling in different directions.

conflict, the selection on females is direct and focused on reducing female mating costs.

Such costs cannot be incorporated into the traditional models of sexual selection developed by R. A. Fisher in the 1930s, which propose a genetic benefit of female mate choice through the enhanced mating ability of sons. In contrast, costs of choice are not a problem for 'good genes' (indicator) models, but these assume that female choice will result in the acquisition of genetic fitness benefits for the offspring, and this is not generally assumed to occur under sexual conflict. The non-adaptive result of female mating biases also distinguishes sexual conflict from direct-benefit models.

Sexual conflict could therefore operate within this traditional sexual-selection framework but be distinguished from it by the nature of the forces acting on female mating biases. However, the authors also suggest that these standard equilibrium models may be inappropriate for analysing sexual conflict. For example, if males had a vast array of ways in which to manipulate females, and females had a similarly large number of ways in which to respond, the dynamics of this contest could well be more suited to non-equilibrium modelling. If this were true, then evolutionary change driven by sexual conflict would be different from any processes of mate choice with which we are familiar.
Arnqvist and Rowe expose the core problem in the empirical study of sexual conflict: it is difficult to observe. It is common to see what looks like overt aggression or fights between male and female mating partners. But such observations by themselves do not necessarily indicate that sexual conflict is responsible and cannot, without further information, exclude the possibility of sexual selection. Arnqvist and Rowe highlight the fact that a process of continual adaptation and counter-adaptation could obscure evolutionary change resulting from sexual conflict. It may also be difficult to measure the costs and benefits of reproductive decisions under an appropriate range of relevant conditions, or even to decide what data are required. As the authors state, "unambiguous experimental data of sexual conflict are really quite scarce".

The best part of the ensuing discussion of the empirical studies in which sexual conflict could be operating covers the species for which good data are available, notably bed bugs, diving beetles, water-striders, fruitflies and dunnocks. The authors also consider numerous further cases in which sexual conflict might be occurring. These are more anecdotal but provide some fascinating natural history, such as the use of gin traps by male sage bush crickets, the 'playing dead'strategy of some species of robber fly, and the male funnel-web spider, which drugs its mate before copulation. This remarkable wealth of examples hints at the potentially ubiquitous distribution and importance of sexual conflict, and represents an extremely valuable resource that should stimulate further study and experimentation on the systems described. Tracey Chapman is in the Department of Biology, University College London, Gower Street, London WC1E6BT, UK. 